\title{
DESENVOLVIMENTO SUSTENTÁVEL E SAÚDE: UMA REVISÃO INTEGRATIVA ${ }^{1}$
}

Roberto Ramon Queiroz de Assis, Universidade Federal de Campina Grande/Centro de

Formação de Professores (UFCG-CFP), E-mail: roberto.ramon9@gmail.com

Leandro Januário de Lima, Universidade Federal de Campina Grande/Centro de Formação de Professores (UFCG-CFP), E-mail: leandrojanuario100@gmail.com

José Ferreira Lima Júnior Universidade Federal de Campina Grande/Centro de Formação de Professores (UFCG-CFP), E-mail: ferreirajunior@cfp.ufcg.edu.br

\section{RESUMO}

O presente trabalho discorre sobre a sustentabilidade e suas relações com o campo da saúde, por meio de uma revisão integrativa da literatura, busca compreender as relações entra as duas áreas com foco em planejamento institucional em saúde. Para a construção da temática e posterior análise, utilizou-se de artigos científicos que foram publicados em periódicos nacionais e internacionais, tendo a busca dos artigos sido feita a partir de descritores constantes nos Descritores em Ciências da Saúde (DeCS). Foram pesquisadas três bases eletrônicas de dados. Adotou-se o método bibliográfico e documental. Esta pesquisa suscita que a sustentabilidade deve ser continuamente reforçada dentro das instituições por meio da educação ambiental e ressalta a importância de cada profissional envolvido na assistência em saúde ser agente promotor do desenvolvimento sustentável nas instituições.

PALAVRAS-CHAVE: Desenvolvimento; Sustentabilidade; Planejamento institucional em saúde.

\section{SUSTAINABLE DEVELOPMENT AND HEALTH: AN INTEGRATING REVIEW}

\begin{abstract}
This work discusses sustainability and its relations with the field of health, through an integrative literature review, seeks to understand relationships enters the two areas focusing on institutional health planning. For the thematic construction and subsequent analysis, it was used for scientific articles that were published in national and international journals and the search for the articles was made from the descriptors in the Descriptors in Health Sciences

\footnotetext{
${ }^{1} \mathrm{O}$ presente trabalho contou com apoio financeiro do conselho nacional de desenvolvimento científico e tecnológico CNPq-Brasil .
} 
(DECS). Three electronic data bases were searched. The bibliographical and documentary method was adopted. This research raises that sustainability must be continually strengthened within the institutions through environmental education and the importance of each professional involved in health care assistance being the promoter of sustainable development in the institutions.

KEYWORDS: Development; Sustainability; Institutional health planning.

\section{DESARROLLO SOSTENIBLE Y SALUD: UNA REVISIÓN INTEGRATIVA RESUMEN}

El presente estudio aborda la sostenibilidad y sus relaciones con el campo de la salud, a través de una revisión integrativa de la literatura, busca entender las relaciones entre las dos áreas con énfasis en la planificación institucional de la salud. Para la construcción de la temática y posterior análisis, se utilizaron artículos científicos que fueron validados y publicados en revistas nacionales e internacionales, la búsqueda de los artículos se realizó a partir de una consulta a los Descriptores en Ciencias de la Salud (DeCS). Se adoptó el método bibliográfico y documental. Esta investigación señala sucintamente que la sostenibilidad debe ser continuamente fortalecida dentro de las instituciones a través de la educación ambiental y destaca la importancia de cada profesional involucrado en la atención de la salud como agentes que promueven el desarrollo sostenible en las instituciones.

PALABRAS CLAVES: Desarrollo; Sostenibilidad; Planificación institucional en salud.

\section{INTRODUÇÃO}

O desenvolvimento sustentável (DS) entra no cenário das políticas públicas com a emergente preocupação do uso do planeta, advinda da necessidade de ofertar bens e serviços a uma população crescente e, por conseguinte, um alerta do crescimento econômico; questões essas bastante difundidas em eventos internacionais como a $\mathrm{RIO}+20$, que discutem os desafios do DS e a implantação de uma economia verde (BUSS, et al 2012).

Sendo assim, o Desenvolvimento sustentável vem ganhando espaço nas diversas áreas de conhecimento e atuação, suscitando em instituições e meio científico, agregar valores da sustentabilidade nos seus processos produtivos, com a finalidade de garantir bens e serviços à população presente e promover a seguridade que populações futuras também tenham o direto de usar dos mesmos recursos (LOURENÇO, et al 2013).

Comumente, o termo sustentabilidade está associado às questões ambientais, no que tange a capacidade de produção e autorreparação dos recursos naturais e ao nível econômico, 
advindo do crescente desenvolvimento social, que impulsiona um consumismo desenfreado, apoiado sobre um modelo de produção capitalista que depende diretamente dos recursos naturais, que por sua vez são finitos, e sua exploração desenfreada não favorece sua reposição. "Ergue-se, assim, a noção de sustentabilidade sobre a percepção da finitude dos recursos naturais e sua gradativa e perigosa depleção" (NASCIMENTO, 2012 p.51).

Fugindo de um conceito menor, o desenvolvimento sustentável em uma perspectiva contemporânea apoia-se em um tripé, no qual a ciência propõe um conceito ampliado que contemple as três dimensões do DS que são as demandas sociais, econômicas e ambientais do desenvolvimento sustentável (BARBOSA, 2008). Remete-nos então a uma perspectiva holística do DS, que é estudá-lo dentro de um contexto maior de modo a possibilitar a compreensão de seus fenômenos em sua totalidade.

Nessa direção, considerando a complexidade dos fenômenos relativos ao DS, neste texto particular interesse será dado à relação dele com o campo sanitário. A saúde cada vez mais tem se aproximado das políticas e ações sustentáveis, sobretudo devido à grande demanda por serviços/ações de saúde e seus altos custos na manutenção e operacionalização de tecnologias. A partir desses constructos, justifica-se o presente estudo que objetivou realizar uma revisão integrativa da literatura sobre a relação do desenvolvimento sustentável com a área da saúde.

\section{MATERIAIS E MÉTODOS}

Optou-se por um estudo bibliográfico na modalidade de revisão integrativa da literatura científica a qual permite uma ampla análise dos estudos que tratam de determinado assunto promovendo uma síntese de trabalhos, possibilitando a identificação de lacunas e a necessidade de novos estudos (ANDRADE et al., 2012). Segundo Sousa e Silva (2010) a revisão integrativa emerge como uma metodologia que proporciona a síntese do conhecimento e a incorporação da aplicabilidade de resultados de estudos significativos na prática.

A construção da revisão integrativa procedeu-se em seis etapas: identificação do tema, formulação da pergunta norteadora, delimitação dos descritores e seleção das bases de dados, estabelecimento de critérios de exclusão e inclusão dos trabalhos, busca da 
amostragem na literatura e pré-seleção dos trabalhos (leitura de resumos e títulos); leitura dos artigos selecionados e, por fim, a interpretação dos resultados.

A questão norteadora que fomentou este estudo foi: Como o conhecimento científico vem abordando a aplicabilidade do desenvolvimento sustentável no contexto da saúde?

Na seleção dos descritores, utilizaram-se os idiomas inglês e português. Ademais, eles foram identificados a partir de consulta aos Descritores em Ciências da Saúde (DeCS), utilizando filtro por palavra ou termo. A estratégia de busca realizada foi controlada e combinada com os caracteres boleanos and e or. A seleção dos artigos foi feita a partir de três bases de dados, conforme a seguinte tabela (tabela 01)

Tabela 01. Descritores utilizados de acordo com bases de dados. Cajazeiras/PB, 2017.

\begin{tabular}{c|l}
\hline BASES DE DADOS & \multicolumn{1}{c}{ DESCRITORES CONTROLADOS } \\
\hline LILACS & (Sustentabilidade OR Sustainable) AND (Planejamento em Saúde OR \\
& Health Planning) \\
& AND (Hospital Planning OR Planejamento Hospitalar) \\
\cline { 2 - 2 } & (Sustentabilidade OR Sustainable) AND (Planejamento em Saúde OR \\
& Health Planning) \\
\hline PubMed & $\begin{array}{l}\text { (Sustentabilidade or Sustainable) and (Planejamento em Saúde or } \\
\text { Health Planning) and (Hospital Planning or Planejamento Hospitalar) }\end{array}$ \\
\hline Scielo & $\begin{array}{c}\text { (Sustentabilidade OR Sustainable) AND (Planejamento em Saúde OR } \\
\text { Health Planning) }\end{array}$ \\
\hline
\end{tabular}

Para os critérios de inclusão, consideraram-se artigos disponíveis eletronicamente na íntegra, que trataram o desenvolvimento sustentável no contexto da saúde com foco em planejamento institucional ou abordaram a relação entre saúde e DS, disponíveis no período de 2006 a 2016 em periódicos nacionais e internacionais, atentando-se ao seguinte critério de exclusão: os artigos repetidos em mais de uma base ou que não enfocaram o assunto de forma contextualizada como proposto na pergunta norteadora foram excluídos.

Para a análise e posterior síntese dos artigos, após aplicação dos critérios de inclusão a amostra final foi composta por nove trabalhos, dos quais se buscou elucidar seus aspectos gerais em resumo analítico.

\section{RESULTADOS E DISCUSSÃO}


Nesta revisão integrativa, analisaram-se nove artigos que foram buscados conforme estratégia de busca suprarreferida e que atenderam aos critérios de inclusão previamente estabelecidos. Na sequência há uma síntese geral dos artigos avaliados dos quais, cinco são brasileiros e os demais são oriundos da Inglaterra, Austrália, Argentina e Itália.

Quanto ao tipo de delineamento metodológico das pesquisas, dos artigos avaliados evidenciou-se que cinco deles compreendem estudo de caso, dos quais dois possuem abordagem qualitativa; outros dois consistem em artigos de reflexão, além de uma revisão não sistemática da literatura.

Dos artigos avaliados, observou-se que a abordagem dada ao desenvolvimento sustentável é evidenciada como uma necessidade de implantação de novas práticas de atuação em saúde. Apenas três estudos denotam sua aplicabilidade dentro de instituições ou a investigam de forma direta (SARI \& CAMPONOGR, 2014; SVALDI. et al.2013; GALLO \& SETTI. 2012); mostrando que o DS tem se apresentado como resultante das pesquisas, porém tem sido tratado de modo complementar às ações propostas (KEMPFER, et al.2010)

Uma resalva fica ao estudo de Sari (2014) que tratou do DS de forma contextualizada abordando planejamento institucional de uma unidade hospitalar, tendo por objetivo estudar os desafios da educação ambiental na ótica dos indivíduos envolvidos no planejamento e execução das ações. Como resultado obteve-se que a educação ambiental abordada dentro das instituições hospitalares como ferramenta possibilita uma conscientização e futura possível mudança de hábitos; apontando que os desafios da educação ambiental na instituição investigada envolveram, principalmente, a carência de uma política ambiental institucional e a formação ambiental fragilizada dos profissionais.

Nesse sentido, verifica-se um aspecto inovador do DS: a exaltação da importância dos profissionais como colaboradores para alcançar o DS institucional. Esta colaboração também foi evidenciada no estudo de Morris et al. (2014), no qual os autores o definem como stakeholder.

Após avaliação dos artigos obtidos em periódicos nacionais e internacionais, constatou-se que o desenvolvimento sustentável não foi alvo direto das investigações, os estudos elucidam aplicação de métodos como informática em saúde, análise comparativa e estudos de casos para implantar novas formas de trabalhos, em alguns pontos exaltam o DS como sendo uma abordagem diferenciada nas pesquisas no campo da saúde, sendo que 
mesmo os estudos apenas tangenciando a temática, eles denotam a importância do desenvolvimento sustentável (TROVATO, 2014; LUNA, et al, 2014; MORRIS, et al. 2014; GÓMEZ, 2014).

\section{CONCLUSÕES}

O desenvolvimento sustentável ainda não encontrou seu espaço no campo sanitário, apesar de se perceber recentemente que os estudos sanitários de caráter interdisciplinar já o apresentam como parte integrante e aplicável na área de saúde.

A interface entre as temáticas da sustentabilidade e da saúde ainda é pouca evidenciada no Brasil, sendo carente o número de publicações que buscam investigar de forma centrada a dinâmica relação entre tais temas. Nesse sentido, é necessário o desenvolvimento de linhas de pesquisa que busquem investigar as conexões interdisciplinares entre o DS e a saúde.

\section{REFERÊNCIAS BIBLIOGRÁFICAS}

ANDRADE, M. et al. Prevenção de reações de pele devido à teleterapia em mulheres com câncer de mama: revisão integrativa. Rev. Latino-Am. Enfermagem [Internet]. maio-jun. 2012. Acesso em: 06/10/2016. Disponível em: http://www.scielo.br/pdf/rlae/v20n3/pt_a24v20n3.pdf.

ARAúJO, G. C., BUENO, M. P., SOUZA, A. A., MENDONÇA, P. S. M. Sustentabilidade empresarial: conceito e indicadores. III CONVIBRA, 2006. Disponível em: http://www.convibra.com.br/2006/artigos/61_pdf.pdf. Acesso em: 06/10/2016.

BARBOSA, G.S. O desafio do desenvolvimento sustentável. Revista Visões, v.1, n.4, 2008.

BUENO, Roberto Eduardo; MOYSES, Simone Tetu; BUENO, Paula Alexandra Reis e MOYSES, Samuel Jorge. Governança, sustentabilidade e equidade no plano de saúde de São 
José dos Pinhais, Brasil. Rev Panam Salud Publica [online]. 2013, vol.34, n.6, pp.416-421. ISSN 1680-5348.

BUSS, et al. Health and environmental governance for sustainable development/Governanca em saúde e ambiente para o desenvolvimento sustentável. 2012. (articulo en português).Ciência \& Saúde Coletiva, 17(6), 1479.

CAMPOS, F. J. B.; RAMOS, H. R. Aplicação do modelo TBL em um hospital público. Journal of Environmental Management and Sustainability - JEMS Revista de Gestão Ambiental e Sustentabilidade - GeAS Vol. 3, N. 1. Jan./ Abr. 2014.

DANIEL LUNA, et al. Health Informatics in Developing Countries: Going beyond Pilot Practices to Sustainable Implementations: A Review of the Current Challenges. Healthc Inform Res. 2014 January;20(1):3-10. http://dx.doi.org/10.4258/hir.2014.20.1.3

GALLO, Edmundo and SETTI, Andréia Faraoni Freitas. Abordagens ecossistêmica e comunicativa na implantação de agendas territorializadas de desenvolvimento sustentável e promoção da saúde. Ciênc. saúde coletiva [online]. 2012, vol.17, n.6, pp.1433-1446. ISSN 1413-8123. http://dx.doi.org/10.1590/S1413-81232012000600008.

GÓMEZ. Proposing a sequential comparative analysis for assessing multilateral health agency transformation and sustainable capacity: exploring the advantages of institutional theory. Globalization and healht 2014 10:38. Doi: 10.1186/1744-8603-10-38.

KEMPFER, Silvana Silveira; BIROLO, Ioná Vieira Bez; MEIRELLES, Betina Homer Schlindwein and ERDMANN, Alacoque Lorenzini. Reflexão sobre um modelo de sistema organizacional de cuidado de enfermagem centrado nas melhores práticas. Rev. Gaúcha Enferm. (Online) [online]. 2010, vol.31, n.3, pp.562-566. ISSN 1983-1447. http://dx.doi.org/10.1590/S1983-14472010000300022.

LOURENÇO, L.; DENISE, C. Sustentabilidade social e desenvolvimento sustentável. RACE, Unoesc, v. 12, n. 1, p. 9-38, jan./jun. 201 Disponível em: http://editora.unoesc.edu.br/index. php/race/article/viewFile/2346/pdf. Acesso em: 06/10/2016. 
MORRIS, et al. Principles to guide sustainable implementation of extended-scope-of-practice physiotherapy workforce redesign initiatives in Australia: stakeholder perspectives, barriers, supports, and incentives. Journal of Multidisciplinary Healthcare 2014:7 249-258.

NASCIMENTO, E.P. Trajetória da sustentabilidade: do ambiental ao social, do social ao econômico. Estud. av. [online]. 2012, vol.26, n.74, pp.51-64. ISSN 0103-4014. http://dx.doi.org/10.1590/S0103-40142012000100005.

SOUZA, M.T. SILVA, M. D.; CARVALHO, R. Revisão integrativa: o que é e como fazer. Einstein (São Paulo), v. 8, p. 102-106, 2010.

SVALDI, Jacqueline Sallete Dei; ZAMBERLAN, Claudia; SIQUEIRA, Hedi Crecencia Heckler de. Abordagem ecossistêmica: uma possibilidade para construir conhecimento sustentavel em enfermagem/saúde. Esc. Anna Nery, Rio de Janeiro , v. 17, n. 3, p. 542-547, ago. 2013 . Disponível em <http://www.scielo.br/scielo.php?script=sci_arttext\&pid=S1414$81452013000300542 \& \operatorname{lng}=\mathrm{pt} \& \mathrm{nrm}=\mathrm{iso}>$. acessos em 28 jun. 2017. http://dx.doi.org/10.1590/S1414-81452013000300019.

TROVATO. Sustainable medical research by effective and comprehensive medical skills: overcoming the frontiers by predictive, preventive and personalized medicine. The EPMA Journal 2014 5:14. doi:10.1186/1878-5085-5-14.

SARI, V; CAMPONOGARA, S. Desafios da educação ambiental em uma instituição hospitalar. Texto Contexto Enferm, abr-jun 2014, 23(2). Disponible en <http://www.indexf.com/textocontexto/2014/23228.php> 